\title{
The mixed-lineage kinase 3 inhibitor URMC-099 facilitates microglial amyloid- $\beta$ degradation
}

Weiguo Dong ${ }^{1,2 \dagger}{ }^{\dagger}$ Christine M. Embury ${ }^{1 \dagger}$, Yaman Lu ${ }^{1 \dagger}$, Sarah M. Whitmire ${ }^{1}$, Bhagyalaxmi Dyavarshetty ${ }^{1}$, Harris A. Gelbard ${ }^{3}$, Howard E. Gendelman ${ }^{1,4}$ and Tomomi Kiyota ${ }^{1 *}$

\begin{abstract}
Background: Amyloid- $\beta$ (A $\beta$ )-stimulated microglial inflammatory responses engage mitogen-activated protein kinase (MAPK) pathways in Alzheimer's disease (AD). Mixed-lineage kinases (MLKs) regulate upstream MAPK signaling that include p38 MAPK and c-Jun amino-terminal kinase (JNK). However, whether MLK-MAPK pathways affect $A \beta$-mediated neuroinflammation is unknown. To this end, we investigated if URMC-099, a brain-penetrant small-molecule MLK type 3 inhibitor, can modulate $A \beta$ trafficking and processing required for generating AD-associated microglial inflammatory responses.

Methods: AB1-42 (Aß42) and/or URMC-099-treated murine microglia were investigated for phosphorylated mitogen-activated protein kinase kinase (MKK)3, MKK4 (p-MKK3, p-MKK4), p38 (p-p38), and JNK (p-JNK). These pathways were studied in tandem with the expression of the pro-inflammatory cytokines interleukin (IL)-1 $\beta$, IL-6, and tumor necrosis factor (TNF)-a. Gene expression of the anti-inflammatory cytokines, IL-4 and IL-13, was evaluated by real-time quantitative polymerase chain reaction. $A \beta$ uptake and expression of scavenger receptors were measured. Protein trafficking was assessed by measures of endolysosomal markers using confocal microscopy.

Results: Aß42-mediated microglial activation pathways were shown by phosphorylation of MKK3, MKK4, p38, and JNK and by expression of IL-1 $\beta$, IL-6, and TNF- $\alpha$. URMC-099 modulated microglial inflammatory responses with induction of IL-4 and IL-13. Phagocytosis of A 42 was facilitated by URMC-099 with up-regulation of scavenger receptors. Co-localization of $A \beta$ and endolysosomal markers associated with enhanced $A \beta 42$ degradation was observed.

Conclusions: URMC-099 reduced microglial inflammatory responses and facilitated phagolysosomal trafficking with associated A degradation. These data demonstrate a new immunomodulatory role for URMC-099 to inhibit MLK and to induce microglial anti-inflammatory responses. Thus, URMC-099 may be developed further as a novel disease-modifying AD therapy.
\end{abstract}

Keywords: Mixed-lineage kinase 3, Alzheimer's disease, Amyloid- $\beta$, Microglia, Phagocytosis, Endolysosomal pathway

\footnotetext{
* Correspondence: tkiyota@unmc.edu

${ }^{\dagger}$ Equal contributors

${ }^{1}$ Department of Pharmacology and Experimental Neuroscience, University of

Nebraska Medical Center, Omaha, NE 68198-5930, USA

Full list of author information is available at the end of the article
} 


\section{Background}

Neuroinflammation is a pathogenic driver for Alzheimer's disease (AD) [1-3]. Amyloid- $\beta(A \beta)$-activated microglia secrete pro-inflammatory neurotoxins that are strongly linked to AD-associated neural injury. Such inflammatory responses are notably tied beyond AD to common age-related neurodegenerative, neuroinfectious, and neuroinflammatory disorders [4-13]. Activated microglia, in turn, stimulate neurons to produce more $A \beta$ and the microtubule-associated protein tau, in a vicious paracrine loop [14]. The events change the brain's microenvironment and further affect microglial activation, leading to progressive neuronal injuries. The end result is a paracrine feedback of neurotoxin amplification that drives disease with $A \beta$ serving as the principal inducer of innate immune activation [15-17]. This has led many researchers to develop the means to harness immunity for therapeutic gain. Notably, $A \beta$ vaccination can effectively clear $A \beta$ in both mouse models of human disease and $\mathrm{AD}$ patients $[18,19]$. For treatment of $\mathrm{AD}, \mathrm{A} \beta$ immunization reduces the A 342 load with immunomodulation, including microglial phagocytosis and changed lysosomal and scavenger markers [20, 21]. However, such immunizations can also result in the development of meningoencephalitis ( 6\%) [22], spongiosis, and neuronal loss [23]. Since removal of plaques at later stages of disease marked by neurofibrillary tangle formation was not proven to be beneficial, alternative treatment strategies are likely required. These might improve clinical outcomes if administered years before clinical signs and symptoms emerge. Indeed, such a therapeutic approach could speed clearance of $A \beta$ and modify inflammatory activities before disease develops.

Prior therapeutic approaches focused on development of immunomodulatory agents that affect AD microglial responses. For example, nonsteroidal antiinflammatory drugs (NSAIDs) or antioxidants reduce harmful microglial inflammatory activities and protect neurons in animal models of human disease [24, 25]. While the drugs are effective in converting microglial polarization from an M1 (classical activation) to an M2 (alternative activation) phenotype and attenuating neurotoxin production, successful human therapeutic translation remains out of reach $[1,25,26]$.

While the "selectively non-selective" brain-penetrant mixed-lineage kinase type 3 (MLK3) inhibitor, URMC099, can attenuate neuroinflammatory responses and facilitate the actions of long-acting nanoformulated antiretroviral drugs during human immunodeficiency virus1 (HIV-1) infection [27-29], it is not known whether it could also harness autophagy. If operative, this could occur as a secondary response to phagocytosis of $A \beta$. Here, we show yet another novel therapeutic role of URMC-099 as an immune modulator in microglial inflammation and phagocytosis through accelerating phagolysosomal pathway
$\mathrm{A} \beta$ degradation. In this manner, we posit that the drug could be developed as a novel AD therapeutic candidate.

\section{Methods \\ Microglia isolation and cultivation}

Housing, care, and breeding of non-transgenic mice (B6.129 hybrid background) were approved by the Institutional Animal Care and Use Committee of the University of Nebraska Medical Center. Primary cultured mouse microglia were prepared from the postnatal day 1 newborn mouse brains [30-32]. Meninges-free newborn mouse cortices were minced and trypsinized, followed by mechanical dissociation and filtration to remove tissue chunks. Cells were plated onto plastic tissue culture bottles as mixed glial cultures in Dulbecco's modified eagle medium supplemented with heat-inactivated $10 \%$ fetal bovine serum, $50 \mu \mathrm{g} / \mathrm{ml}$ penicillin/streptomycin (Life Technologies, Carlsbad, CA, USA) and macrophage colony-stimulating factor (MCSF). Microglia were released from astrocytes in the tissue culture media by shaking. Non-adherent cells were collected 7-14 days after plating.

\section{$A \beta$ activation and URMC-099 treatment}

Microglia were plated at a density of $2 \times 10^{5}$ onto 24-well plates for RNA/protein extraction or onto 24-well plates with glass coverslips for confocal microscopy. $2 \times 10^{4}$ cells were placed in each 96-well plate for immunofluorescence studies. One day before A $\beta$ exposure and URMC-099 treatment, media was replaced without MCSF. Microglia were pre-incubated for $30 \mathrm{~min}$ with URMC-099 (100 nM) [28]. Notably, URMC-099 administered at $100 \mathrm{nM}$ did not affect microglial viability as measured by the 3-(4,5-dimethylthiazol-2-yl)-2,5-diphenyltetrazolium bromide (MTT) assay (Additional file 1: Figure S1) that was performed as previously described [33]. After pre-incubation with URMC-099, microglia were incubated with media containing the monomeric A $342(10 \mu \mathrm{M}$, Life Technologies, Carlsbad, CA, USA) prepared as previously described [32]. Since monomeric rather than oligomeric A $\beta 1-42$ (A 342 ) peptides are effectively phagocytosed by human monocyte-derived macrophages [34], monomeric A 342 was used. After $A \beta$ treatment, microglia were harvested for total RNA or protein extraction or fixed with freshly depolymerized $4 \%$ paraformaldehyde (PFA) in PBS for $15 \mathrm{~min}$ for immunofluorescence assays.

\section{Immunoblot and ELISA tests}

Microglial cells were harvested using ice-cold RIPA buffer with protease and phosphatase inhibitor cocktail at end points and centrifuged at $15,000 x \mathrm{~g}$ for $10 \mathrm{~min}$ at $4{ }^{\circ} \mathrm{C}$ to collect cell lysates. Protein concentrations were determined using a Micro BCA Protein Assay (Thermo Fisher Scientific, Waltham, MA, USA). For immunoblots, protein lysates were diluted 1:1 with Laemmli buffer containing 
$\beta$-mercaptoethanol, incubated at $100{ }^{\circ} \mathrm{C}$ for $5 \mathrm{~min}$, electrophoresed on sodium dodecyl sulfate-polyacrylamide gels, and electroblotted to $0.45-\mu \mathrm{m}$ pore size polyvinylidene fluoride membranes (Immobilon-P, Millipore, Billerica, MA, USA). Membranes were blocked in $3 \%$ bovine serum albumin/TBST or $5 \%$ skim milk/TBST and incubated with antibodies (Abs) to phospho-MKK3 (p-MKK3), total MKK3, phospho-MKK4 (p-MKK4), total MKK4, phosphop38 (p-p38), total p38, phosphor-JNK (p-JNK), total JNK (1:1000, Cell Signaling Technology, Danvers, MA), CD36, CD47 (1:200, Santa Cruz Biotechnology, Santa Cruz, CA, USA), and $A \beta$ (6E10, 1:1000, Covance, Emeryville, CA, USA), at $4{ }^{\circ} \mathrm{C}$ for overnight, followed by 30 -min incubation in $3 \%$ bovine serum albumin/TBST or $5 \%$ skim milk/ TBST with HRP-conjugated anti-goat, mouse, or rabbit IgG Abs (1:2000, Santa Cruz Biotechnology, Santa Cruz, CA, USA). Immunoreactive bands were detected with SuperSignal West Pico or Femto Chemiluminescent substrate and captured using a myECL Imager (Thermo Fisher Scientific, Waltham, MA, USA). After detection of the bands, membranes were incubated with Restore Western Blot Stripping Buffer (Thermo Fisher Scientific, Waltham, MA, USA) and were then used to detect $\beta$-actin for normalization using HRP-conjugated anti- $\beta$-actin monoclonal (Sigma, St. Louis, MO, USA). For quantitative analysis, ImageJ software (NIH, Bethesda, MD, USA) was used to quantify band intensities relative to total proteins or control $\beta$-actin expression. For ELISA, tissue culture media were subjected to capture antibodies for IL-1 $\beta$, IL-6, and TNF- $\alpha$ (PeproTech, Rocky Hill, NJ, USA) according to the manufacturer's instructions. For A $\beta 42$ ELISA, cell lysates and tissue culture media were subjected to A 422 ELISA (Life Technologies, Carlsbad, CA, USA) according to the manufacturer's instructions.

\section{Immunofluorescence and confocal microscopy}

Immunofluorescence was performed using pan- $\mathrm{A} \beta \mathrm{Ab}$ (rabbit polyclonal, 1:100) and Alexa Fluor ${ }^{\circ} 88$-conjugated anti-rabbit IgG (1:1000), followed by 30 min counterstaining with 4',6-diamino-2-phenyl-indole (DAPI) (all from Life Technologies, Carlsbad, CA, USA). Images were captured using DP Controller and DP Manager with a DP71 digital camera (Olympus, Orangeburg, NY, USA) attached to a Nikon Eclipse TE300 inverted microscope (Nikon, Melville, NY, USA). For confocal microscopy, PFAfixed microglia were immunostained with Abs to $A \beta$ (6E10, 1:1000, Covance, Emeryville, CA, USA), Rab5, Rab7 (rabbit polyclonal, 1:500, Santa Cruz Biotechnology, Santa Cruz, CA, USA), and Lamp1 (rabbit polyclonal, ab24170, 1:500, Abcam, Cambridge, MA, USA). Alexa Fluor 488 goat antimouse IgG and Alexa Fluor 568 goat anti-rabbit IgG were used as secondary, followed by 30-min counterstaining with DAPI (Life Technologies, Carlsbad, CA, USA). Images were captured using a $63 \mathrm{X}$ oil lens on a LSM 710 confocal microscope (Carl Zeiss Microimaging Inc., Thornwood, NY, USA). Images were quantified using ImageJ software with a co-localization plugin (https://imagej.nih.gov/ij/ plugins/colocalization.html) (NIH, Bethesda, MD, USA).

\section{RNA extraction and transcript analyses}

Total RNA was extracted from microglia using TRIzol (Life Technologies, Carlsbad, CA, USA). For PCR-based gene expression analyses, cDNA was synthesized with $1 \mu \mathrm{g}$ of total RNA as a template using a Verso cDNA synthesis kit (Thermo Fisher Scientific, Waltham, MA, USA), and quantitative real-time RT-PCR (RT2-qPCR) was performed on a thermocycler (Mastercycler Gradient, Eppendorf Scientific Inc., Westbury, NY, USA) using 2x SYBR Green qPCR Master Mix (Biotool.com, Houston, TX, USA) and gene specific primer sets (Table 1). All primer sequences were obtained from PrimerBank (https://pga.mgh.harvard.edu/primerbank/) [35]. Thermal cycler conditions were as follows: $10 \mathrm{~min}$ at $95{ }^{\circ} \mathrm{C}$ for activation of polymerase, followed by 40 cycles of a two-step PCR $\left(95^{\circ} \mathrm{C}\right.$ for $15 \mathrm{~s}$ and $60^{\circ} \mathrm{C}$ for $1 \mathrm{~min}$ ). Relative expression for target genes was determined by the $\Delta \Delta \mathrm{Ct}$ method and normalized with glyceraldehyde-3phosphate dehydrogenase (Gapdh) gene expression as an internal control. Each $\Delta \mathrm{Ct}$ value was determined by subtracting Gapdh $\mathrm{Ct}$ value from the target gene $\mathrm{Ct}$ value. The $\Delta \Delta \mathrm{Ct}$ was calculated by subtracting the $\Delta \mathrm{Ct}$ value of the control from the $\Delta \mathrm{Ct}$ value of other groups. $2^{-\Delta \Delta \mathrm{Ct}}$ represented the average relative amount of mRNA to control for each target gene.

\section{Statistical analyses}

All data were normally distributed and presented as mean values \pm standard errors of the mean (SEM). In the case of single mean comparison, data were analyzed by Student's $t$ test. In case of multiple mean comparisons, the data were analyzed by one-way ANOVA. When there were significant differences between $\geq 3$ sample means, post hoc comparisons with the Newman-Keuls method was performed using statistics software (Prism 4.0, Graphpad Software, San Diego, CA, USA). A value of $p<0.05$ was regarded as a significant difference.

Table 1 Primers for qPCR analysis

\begin{tabular}{|c|c|c|}
\hline Genes & Forward $\left(5^{\prime}-3^{\prime}\right)$ & Reverse $\left(5^{\prime}-3^{\prime}\right)$ \\
\hline$-1 \beta$ & GTGTCTITCCCGTGGACCTTC & CGGAGCCTGTAGTGCAGTTG \\
\hline$L-6$ & CTACCCCAATTCC & TTGGTCCTTAGCCACTCCTTC \\
\hline NF- $a$ & ACTCC & $\mathrm{CC}$ \\
\hline L-4 & GGTCTCAACCCCCAGCTAGT & GCCGATGATCTCTCTCAAGTGAT \\
\hline$L-13$ & CCTGGCTCTTGCTTGCCTT & GGTCTTGTGTGATGTTGCTCA \\
\hline
\end{tabular}




\section{Results}

URMC-099 inhibits microglial p38 and JNK MAPK signaling cascades

A $\beta$ activates p38 and JNK MAPK cascades to produce pro-inflammatory mediators in microglia [36-38], and has previously been shown to activate MLK3 in cortical neurons [39], but not microglia. Thus, we tested whether URMC-099 could affect the MAPK cascades in A 342 stimulated microglia (Fig. 1). As MKK3/6 and MKK4/7 regulate p38 and JNK phosphorylation downstream MLK3 [40], we examined the phosphorylation patterns of MKK3 and MKK4 (Fig. 1a, c). A 30-min A $\beta 42$ treatment increased MKK3 phosphorylation $32.1 \%$ as compared to untreated controls (Fig. 1b). Moreover, trends in MKK4 phosphorylation were also seen with an $8.0 \%$ increase when compared to untreated controls (Fig. 1d). However, URMC-099 treatment significantly reduced both MKK3 and MKK4 phosphorylation in the A $\beta$-stimulated microglia with decreases of 26.9 and $20.5 \%$ in MKK3 and MKK4 phosphorylation, respectively (Fig. 1b, d). The phosphorylation of p38 and p46/ p54-JNK downstream MKK3 and MKK4 was also assayed (Fig. 1e, g). While a 30-min exposure to A $\beta 42$ showed a rapid induction of both p38 and p46/p54-JNK phosphorylation (increases of 84.8, 18.7, and $25.1 \%$ in p38, p46-JNK, and p54-JNK, respectively, as compared to untreated controls, Fig. 1f, h, i), URMC-099 treatment resulted in significant reduction in p38 and JNK phosphorylation in the $\mathrm{A} \beta$-stimulated microglia. Here, decreases were seen at 21.5, 17.3, and $23.7 \%$ in p38, p46-JNK, and p54-JNK, respectively (Fig. 1f, h, i). To assess the effects of URMC-099 on Aß42-mediated microglial cytotoxicity, we measured cell viability using the MTT assay. A 342 -treatment reduced cell viability (Additional file 2: Figure S2) as observed previously [31]. Although URMC-099 treatment did not ameliorate A 342 -mediated cytotoxicity, trends in protection of cell viability were observed with 9.4 and $16.0 \%$ increase in 3- and 48-h incubation, respectively, when compared to A $\beta 42$ treatment (Additional file 2: Figure S2). These results support the notion that URMC-099 inhibits MLK3-MKK3/4-mediated activation of p38 and JNK MAPK cascades in A $\beta$-activated microglia.

\section{URMC-099 inhibits activated microglial pro-inflammatory cytokines}

URMC-099 exerts anti-inflammatory effects on microglia exposed to the HIV-1 Tat [28]. To determine whether URMC-099 has similar effects for $\beta$-amyloidosis, cultured murine microglia were treated with URMC-099 and exposed to soluble $A \beta 42$ for 30 min. While $A \beta 42$ stimulation induced IL-1 $\beta$, IL- 6 , and TNF- $\alpha$ gene expression, URMC-099 reversed this induction (decreases of 84.8, 85.9, and $97.4 \%$ to $A \beta 42$ stimulation for IL-1 $\beta$, IL-6, and TNF- $\alpha$, respectively, Fig. 2a). The qPCR results were validated by measuring protein production of pro-

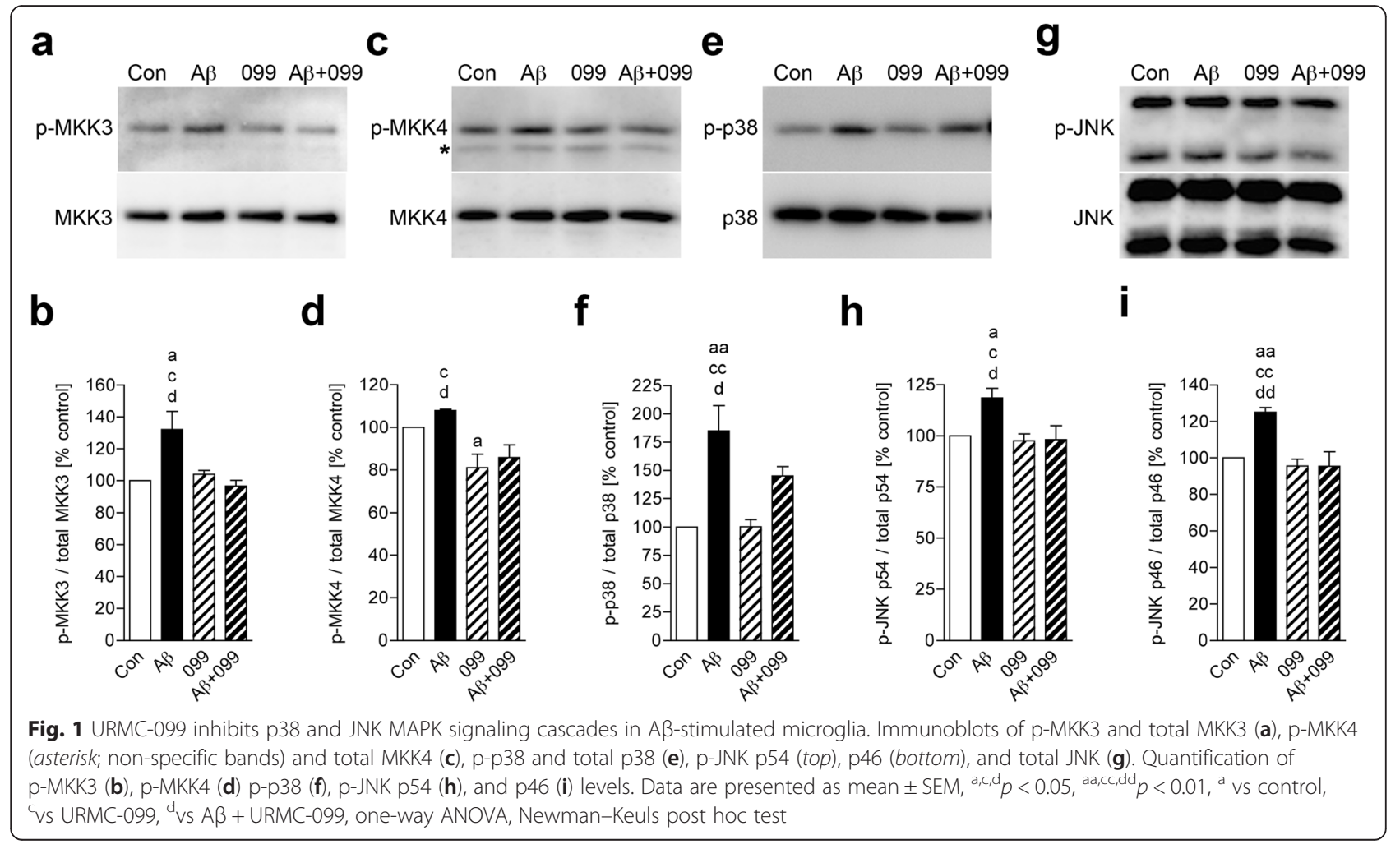




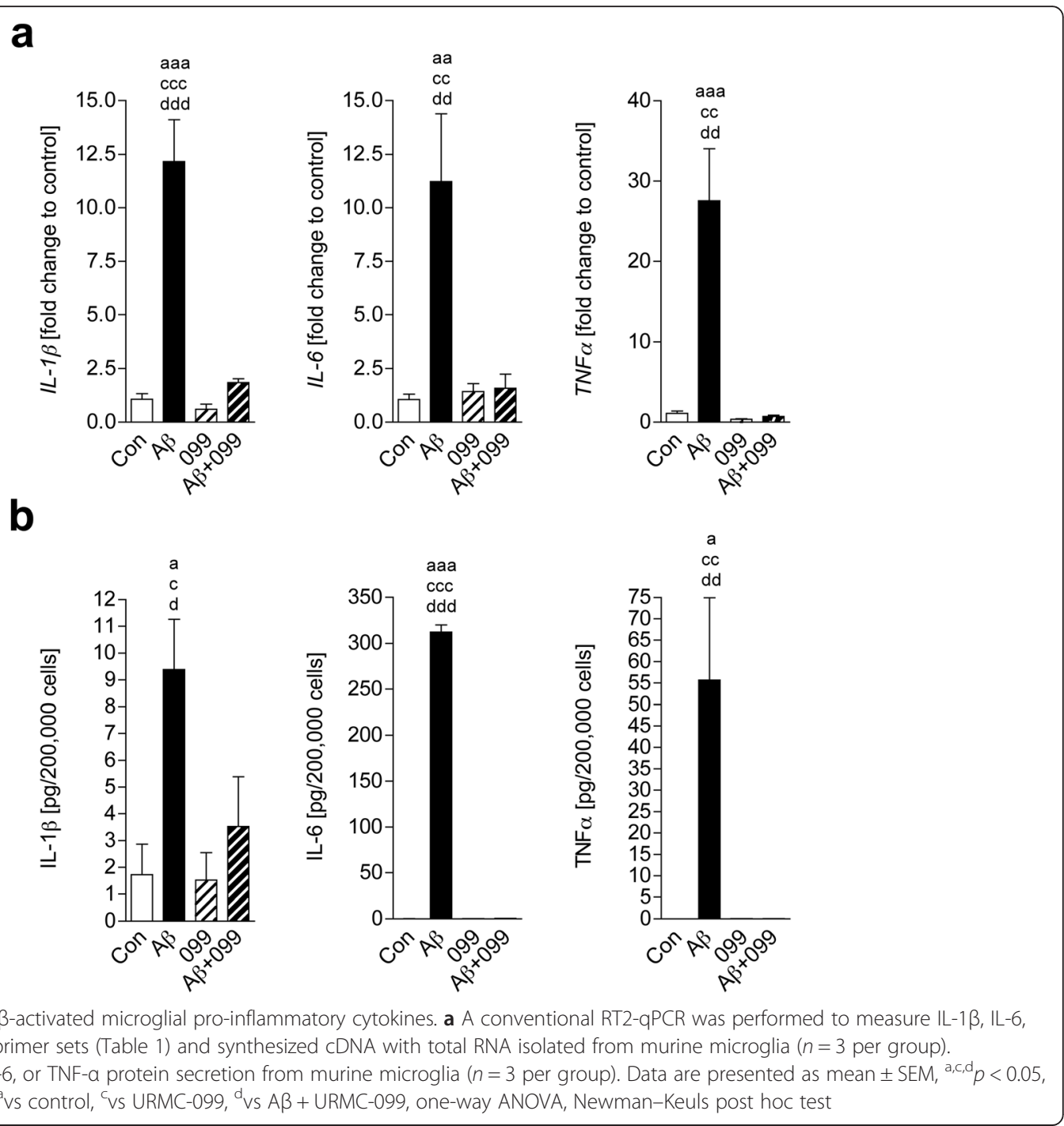

inflammatory cytokines in media. After 48-h A 42 stimulation, URMC-099 treatment inhibited IL-1 $\beta$, IL-6, and TNF- $\alpha$ (decreases of $62.3,99.8$, and $99.9 \%$ to A $\beta 42$ stimulation for IL-1 $\beta$, IL-6, and TNF- $\alpha$, respectively, Fig. $2 b$ ). These data strongly support the anti-inflammatory effects of URMC-099 on A $\beta$-stimulated microglia.

\section{Anti-inflammatory effects of URMC-099 in A 342 -stimulated microglia}

To determine the neuroinflammatory phenotype in URMC-099-treated microglia, total RNA was isolated and RT2-qPCR was performed for genes specific to IL-4 and IL-13. Data were shown as fold change compared to control (Fig. 3). While 30-min A $\beta 42$ stimulation did not alter gene expression of IL-4 and IL-13, URMC-099 significantly induced this expression pattern (increases of 111.1 and $345.7 \%$ in IL-4 and IL-13, respectively, Fig. 3), further supporting the anti-inflammatory effects of URMC-099 on A $\beta$ stimulated microglia.

\section{URMC-099 facilitates microglial $A \beta$ uptake}

Since URMC-099 has anti-inflammatory effects in A $\beta$-stimulated microglia, we hypothesized that cotemporaneous administration of URMC-099 to microglia could accelerate $A \beta$ phagocytosis and subsequent degradation [41-44]. To this end, we tested if URMC099 could facilitate A 342 internalization. URMC-099treated microglia were incubated with $A \beta 42$ for $30 \mathrm{~min}$, then immunostained using pan-A $\beta$ primary and Alexa 488-secondary antibodies (Fig. 4a). Quantification of immunofluorescent intensity showed that relative immunofluorescence was increased in URMC-099-treated microglia, as compared to A $\beta 42$ treatment only $(76.9 \%$ increase, Fig. $4 \mathrm{~b})$, suggesting that URMC-099 promotes $\mathrm{A} \beta$ phagocytosis. 

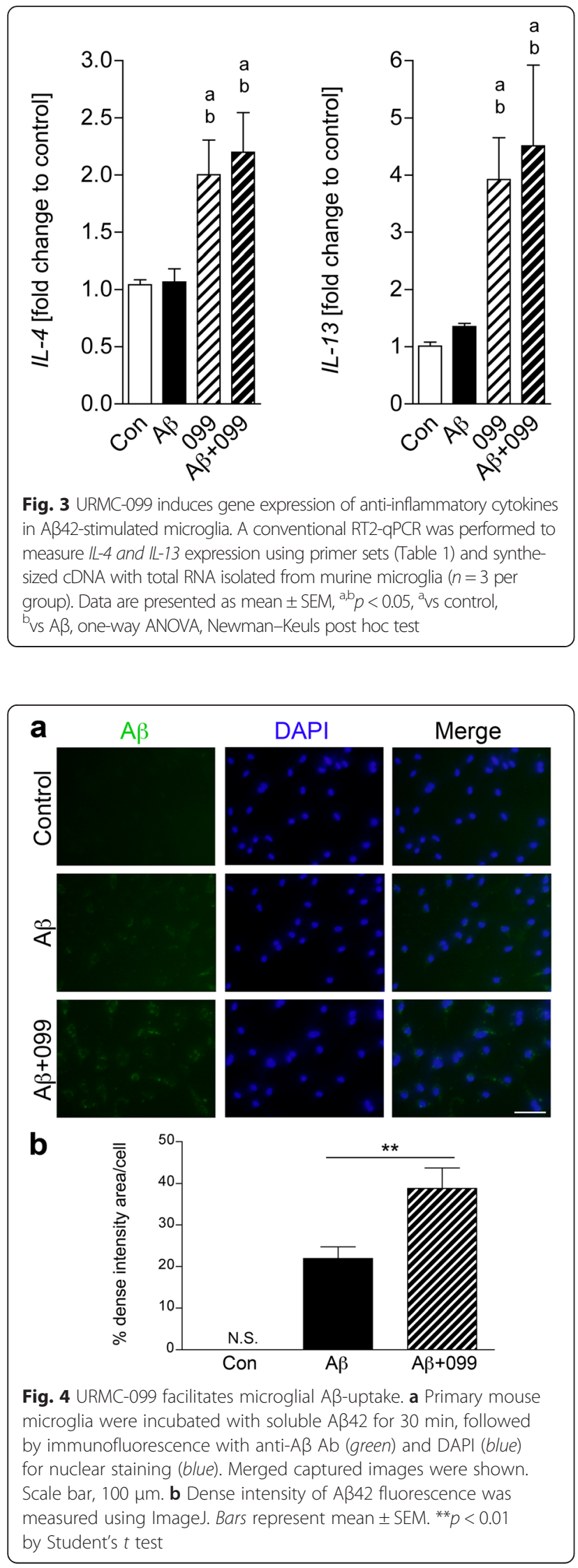

URMC-099 alters scavenger receptor expression in microglia

A previous study demonstrated that microglial phagocytic capacity is linked to scavenger receptor (SR) expression levels in microglia in AD mouse models [45]. To pursue the mechanism of how URMC-099 facilitates A $\beta$ phagocytosis, we examined the expression of SRs. CD36 and CD47 expression was assessed by immunoblotting (Fig. 5a). CD36 expression was increased in all treated groups (increases of 44.4, 56.1, and $44.1 \%$ in URMC-099 only, A $\beta$ only, and A $\beta$ plus URMC-099 to control, respectively, Fig. 5b), while CD47 expression was significantly increased with URMC-099 treatment (increases of 55.9 and $50.2 \%$ in URMC-099 only and A $\beta$ plus URMC-099 to control, respectively, Fig. 5b), demonstrating URMC-099-specific alteration in SR expression.

\section{URMC-099 increases $A \beta$ co-localization with Rab7 and Lamp1}

In response to $A \beta$ binding to SRs, microglia start to engulf $A \beta$ by phagocytosis, and then $A \beta$ enters into the endolysosomal pathway. Thus, we investigated how URMC-099 affects the endolysosomal trafficking underlying $\mathrm{A} \beta$ phagocytosis. Microglia treated as described above were immunostained with antibodies to Rab7 (for late endosomes, Fig. 6a) and Lamp1 (for lysosomes, Fig. $6 \mathrm{c}$ ) at 1 -h post-incubation with $A \beta$. Confocal microscopy demonstrated that co-localization of Rab7 and Lamp1 with A 442 was increased in URMC-099-treated microglia, compared to untreated microglia (21.2 and $26.3 \%$ increases in Rab7 and Lamp1, respectively, Fig. $6 \mathrm{c}, \mathrm{d})$. To investigate $\mathrm{A} \beta$ metabolism, microglia were exposed to $A \beta 42$ for $30 \mathrm{~min}$, washed and cultured in fresh media for additional $1 \mathrm{~h}$, then harvested for immunoblotting (Fig. 7a). Co-treatment with URMC-099 significantly reduced immunoreactivity of monomeric, dimeric, and high molecular weight (HMW) A 342 , as compared to A $\beta 42$ treatment only $(39.3,30.6$, and $42.3 \%$ decreases in monomeric, dimeric, and HMW A $\beta 42$, respectively, Fig. 7b). To validate these results, microglia were exposed to $A \beta 42$ for $30 \mathrm{~min}$, followed by wash and culture in fresh media for additional $1 \mathrm{~h}$, and then $A \beta 42$ release and retention in microglia were quantified using ELISA. While URMC-099 had no effect on A 342 release (Fig. 7c), co-treatment with URMC-099 significantly reduced A $\mathrm{A} 42$ with a $50.2 \%$ reduction (Fig. 7d). These data suggest that URMC-099 promotes microglial endolysosome-mediated degradation.

\section{Discussion}

Microglia are the resident immune cells that serve as the first line of immune defense against invading pathogens in the CNS. The immune response is accelerated by the cell's abilities to recognize pathogen-associated microbial 

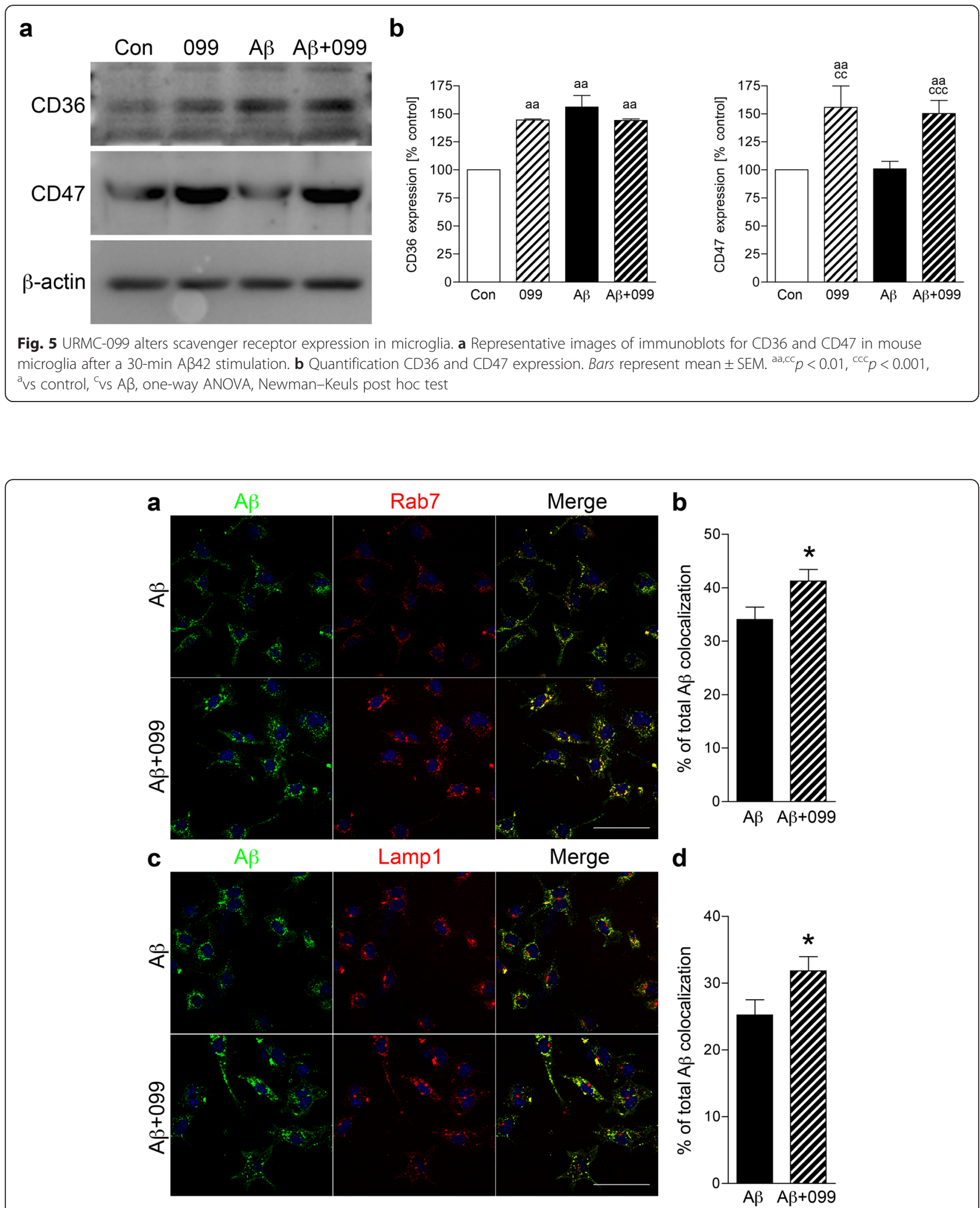

Fig. 6 URMC-099 facilitates subcellular co-localization of A 42 with Rab7 and Lamp1 in murine microglia. Confocal microscopy shows cellular localization of Rab7 late endosomal component (a, red) or Lamp1 lysosomal compartment (b, red) and Aß42 (green). Merged images of Rab7 and Lamp1 are shown. A 42 co-localization with Rab7 (c) or Lamp1 (d) was quantified by ImageJ with a co-localization plugin. Scale bar $=50 \mu \mathrm{m}$. Bars represent mean \pm SEM. ${ }^{*} p<0.05$ by Student's $t$ test 


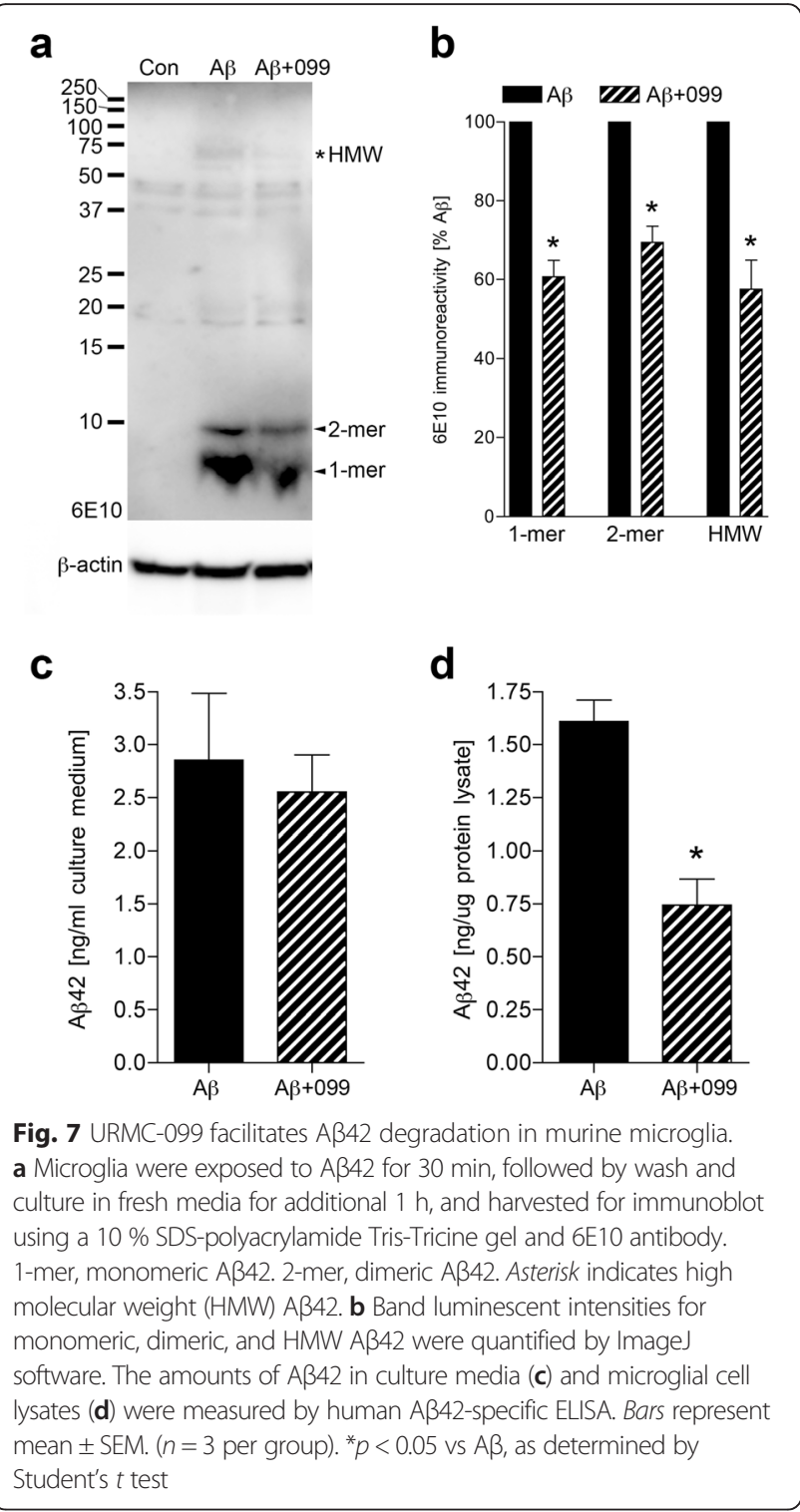

patterns, induce key co-stimulatory molecules, and secrete cytokines that facilitate induction of the adaptive immune response in disease [46-48]. A principal microglial function is to act as scavenger cells to first phagocytose then clear substances such as $A \beta$ [48-50]. Notably, $A \beta$ is considered to be the initiating factor in AD inducing neuroinflammation, subsequent synaptic and axonal injuries, tau hyperphosphorylation, and ultimately neuronal death $[15-17,51]$. With these in mind, a number of therapeutic strategies have been developed for facilitating A $\beta$ removal with the goal of improving disease outcomes. Indeed, anti-inflammatory drugs, amyloid degradation enzymes, and active/passive immunizations have been evaluated as treatment approaches [52-55]. In particular, NSAIDs and antioxidants are effective not only in attenuating the neurotoxins secreted by $A \beta$-stimulated microglia but also in facilitating microglial phagocytic activity for $A \beta$ clearance in animal models of human disease $[1,25,26]$. To date, there has been no clear validation of any of these approaches for disease-modifying outcomes in patients with $\mathrm{AD}$, thus further studies for alternative disease-modifying strategies are warranted. Hence, we investigated whether URMC-099 could ameliorate $A \beta$-mediated microglial activation.

URMC-099 was discovered as a result of a large screening for MLK3 inhibitors structurally containing a pyrrolopyridine scaffold with an aryl piperazine side chain $[28,29]$; it can modify the production of pro-inflammatory mediators by inhibiting MAPK signaling cascades [36-38]. MLKs are MAPK kinase kinases (MAPKKKs) that function as bonafide serine/threonine kinases, although their catalytic domains have features of both serine/threonine and tyrosine kinases. MLKs regulate the p38/JNK signaling cascades that coordinate and orchestrate numerous immune processes [40]. URMC099 is an inhibitor specific to MLK3 $[28,29]$ that is one of the most widely expressed members of the MLK family [56]. MLK3 is expressed in immune effector cells including microglia in the CNS and is activated by cellular and metabolic stress [28, 57-60]. Based on these findings, we posit that URMC-099 is a potential candidate for AD therapeutics. Although it was previously unclear whether $\mathrm{A} \beta$ induces classical microglial activation through MLKMAPK pathways, we showed that URMC-099 reduces phosphorylation of MKK3/MKK4 and p38/JNK and partially protected $\mathrm{A} \beta$-mediated microglial cytotoxicity, suggesting its therapeutic effect targeting MLK-MKKMAPK pathways in $A \beta$-mediated neuroinflammation in microglia.

In $\mathrm{AD}$, microglia contribute to tissue injury through eliciting neuroinflammation and changing the CNS microenvironment. Like peripheral macrophages, microglia can be polarized into M1 and M2 phenotypes based on their functional properties $[25,26,61-63]$. A $\beta$ activation of microglia is associated with production of pro-inflammatory cytokines (IL-1 $1 / / \mathrm{IL}-6 / \mathrm{TNF}-\alpha$ ), traits associated closely with the M1 phenotype $[7,8,11,25,47$, 64]. Alternatively, polarization to an M2 phenotype can initiate an anti-inflammatory and repair phase $[1,25,26]$. The normal homeostatic balance between M1 and M2 phenotypes seems to be disturbed during disease progression in $\mathrm{AD}$ with the presence of more $\mathrm{M} 1$ microglia appearing coincident with aging and disease [26]. Herein, URMC-099 treatment was shown to inhibit IL-1//IL-6/ TNF- $\alpha$ expression and coordinate with the up-regulation of IL-4 and IL-13 genes. These data suggest that URMC099 treatment may initiate phenotypic changes by inducing anti-inflammatory cytokines, thus has neuroprotective activities [42-44]. 
Additionally, microglia in the M2 phase speed $A \beta$ phagocytosis and degradation without neurotoxin production [41-44]. An increase in phagocytosis of $A \beta$ was observed with URMC-099 treatment. Microglial phagocytic capacity is linked to SR expression levels [45]. CD36 is one of the SRs for $A \beta$ and regulates $A \beta$ clearance as well as brain inflammation [65]. While $A \beta$ exposure increased CD36 expression similar to a previous study with BV-2 microglia [66], co-administration of URMC-099 with $A \beta$ failed to reverse this up-regulation. In contrast, URMC-099 increases CD47 expression in microglia both alone and with $A \beta$. Since CD47 is an integrin-associated transmembrane protein and participates in $A \beta$ uptake and microglial pro-inflammatory responses by forming the receptor complex CD36/ CD47/ $66 \beta 1$ integrin, which in turn stimulates microglial phagocytic activity [67-69]. By increasing CD47 activity, our results suggest that URMC-099 facilitates CD36/ CD47/ 6 631-integrin-mediated microglial phagocytic activity of A $\beta$. Moreover, URMC-099 facilitates colocalization of Rab7 and Lamp1 with A 42 , which may promote endolysosomal-mediated $\mathrm{A} \beta$ degradation and metabolism. Our data in aggregate suggests that these pathologic events can be altered with the challenge of how and when to optimally use disease-modifying agents such as URMC-099 to restore homeostasis to the brain's microenvironment.

\section{Conclusions}

URMC-099 inhibits A $\beta$-mediated phosphorylation of MKK3/4-p38/JNK and pro-inflammatory responses, and up-regulates phagolysosomal trafficking and degradation of A $\beta$. Such inhibition of phosphorylation by URMC-099 correlates with inhibition of microglial activation. URMC099 serves as an immune modulatory and neuroprotective agent that may be developed to combat AD.

\section{Additional files}

Additional file 1: Figure S1. URMC-099 treatment for 3, 24, or $48 \mathrm{~h}$ has no effect on microglial viability by using the 3-(4,5-dimethylthiazol-2-yl)2,5-diphenyltetrazolium bromide assay. Data are presented as mean \pm SEM. (TIF $1588 \mathrm{~kb}$ )

Additional file 2: Figure S2. MTT assay of microglia treated with A $\beta 42$ and URMC-099. Data are presented as mean \pm SEM. (TIF 613 kb)

\section{Abbreviations}

$A b$, antibody; AD, Alzheimer's disease; ANOVA, analysis of variance; $A \beta$, amyloid- $\beta$; $B C A$, bicinchoninic acid assay; $C D$, cluster of differentiation; CDNA, complementary deoxyribonucleic acid; CNS, central nervous system; DAPI, diamino-2-phenyl-indol; ELISA, enzyme-linked immunosorbent assay; Gapdh, glyceraldehyde-3-phosphate dehydrogenase; HRP, horseradish peroxidase; IgG, immunoglobulin G; IL, interleukin; JNK, c-Jun amino-terminal kinase; Lamp1, lysosomal-associated membrane protein 1; MAPK,

mitogen-activated protein kinase; MCSF, monocyte colony-stimulating factor; MKK, mitogen-activated protein kinase kinase; MLK, mixed-lineage kinase; NSAID, nonsteroidal anti-inflammatory drug; PCR, polymerase chain reaction;
PFA, paraformaldehyde; RIPA, radioimmunoprecipitation assay buffer; RNA, ribonucleic acid; SR, scavenger receptor; TBST, Tris-buffered saline containing $0.1 \%$ Tween-20; TNF-a, tumor necrosis factor-a

\section{Acknowledgements}

The authors thank James R. Talaska and Janice A. Taylor (Confocal Laser Scanning Core facility, University of Nebraska Medical Center) for assistance with confocal microscopy.

\section{Funding}

This work was supported in part by NIH Grants AG043540, DA028555, NS036126, NS034239, MH064570, NS043985, and MH062261 and DOD Grant 421-20-09A to HEG, the Carol Swarts Emerging Neuroscience Fund, start-up funds from the Department of Pharmacology and Experimental Neuroscience, and the Shoemaker Award for Neurodegenerative Research to TK.

\section{Availability of data and materials}

The datasets supporting the conclusions of this article are included within the article and its additional file.

\section{Authors' contributions}

$W D, C M E$, and $Y L$ performed the experiments and analyzed the data. SW and BD performed the experiments and assisted in the data analysis. HAG provided the URMC-099 for study, provided experimental suggestions, and assisted in writing the manuscript. HEG supervised the research and wrote the manuscript. TK supervised, designed the research, analyzed the data, and wrote the manuscript. All authors discussed the results and conclusions, reviewed, and commented the manuscript. All authors read and approved the final manuscript.

\section{Competing interests}

The authors with the exception of HAG and HEG declare that they have no competing interests. HAG and HEG are members of the scientific advisory board of WavoDyne Therapeutics, Inc., which holds the exclusive license for URMC-099.

\section{Consent for publication}

Not applicable.

\section{Ethics approval and consent to participate}

Housing, care, breeding of non-transgenic mice, and microglial isolation were approved by the Institutional Animal Care and Use Committee at the University of Nebraska Medical Center.

\section{Author details}

${ }^{1}$ Department of Pharmacology and Experimental Neuroscience, University of Nebraska Medical Center, Omaha, NE 68198-5930, USA. ²Department of Integrated Traditional Chinese and Western Medicine, Fujian University of Traditional Chinese Medicine, Fuzhou, Fujian 350122, People's Republic of China. ${ }^{3}$ Department of Neurology, Center for Neural Development \& Disease, School of Medicine and Dentistry, University of Rochester Medical Center, Rochester 14642, NY, USA. ${ }^{4}$ Department of Internal Medicine, University of Nebraska Medical Center, Omaha 68198-5880, NE, USA.

Received: 17 May 2016 Accepted: 27 June 2016

Published online: 11 July 2016

\section{References}

1. Heneka MT, Carson MJ, El Khoury J, Landreth GE, Brosseron F, Feinstein DL, Jacobs AH, Wyss-Coray T, Vitorica J, Ransohoff RM, et al. Neuroinflammation in Alzheimer's disease. Lancet Neurol. 2015;14:388-405.

2. Villegas-Llerena C, Phillips A, Garcia-Reitboeck P, Hardy J, Pocock JM. Microglial genes regulating neuroinflammation in the progression of Alzheimer's disease. Curr Opin Neurobiol. 2015;36:74-81.

3. Heppner FL, Ransohoff RM, Becher B. Immune attack: the role of inflammation in Alzheimer disease. Nat Rev Neurosci. 2015;16:358-72.

4. Lee JK, Tran T, Tansey MG. Neuroinflammation in Parkinson's disease. J Neuroimmune Pharmacol. 2009;4:419-29.

5. Zhao W, Beers DR, Appel SH. Immune-mediated mechanisms in the pathoprogression of amyotrophic lateral sclerosis. J Neuroimmune Pharmacol. 2013;8:888-99. 
6. Hong S, Banks WA. Role of the immune system in HIV-associated neuroinflammation and neurocognitive implications. Brain Behav Immun. 2015;45:1-12.

7. Szczepanik AM, Funes S, Petko W, Ringheim GE. IL-4, IL-10 and IL-13 modulate A beta(1-42)-induced cytokine and chemokine production in primary murine microglia and a human monocyte cell line. J Neuroimmunol. 2001;113:49-62.

8. Parvathy S, Rajadas J, Ryan H, Vaziri S, Anderson L, Murphy Jr GM. Abeta peptide conformation determines uptake and interleukin-1alpha expression by primary microglial cells. Neurobiol Aging. 2009;30:1792-804.

9. Neher JJ, Neniskyte U, Zhao JW, Bal-Price A, Tolkovsky AM, Brown GC. Inhibition of microglial phagocytosis is sufficient to prevent inflammatory neuronal death. J Immunol. 2011;186:4973-83.

10. Murgas P, Godoy B, von Bernhardi R. Abeta potentiates inflammatory activation of glial cells induced by scavenger receptor ligands and inflammatory mediators in culture. Neurotox Res. 2012;22:69-78.

11. Taneo J, Adachi T, Yoshida A, Takayasu K, Takahara K, Inaba K. Amyloid beta oligomers induce interleukin-1beta production in primary microglia in a cathepsin B- and reactive oxygen species-dependent manner. Biochem Biophys Res Commun. 2015;458:561-7.

12. Qin L, Liu Y, Cooper C, Liu B, Wilson B, Hong JS. Microglia enhance beta-amyloid peptide-induced toxicity in cortical and mesencephalic neurons by producing reactive oxygen species. J Neurochem. 2002;83:973-83

13. Szaingurten-Solodkin I, Hadad N, Levy R. Regulatory role of cytosolic phospholipase A2alpha in NADPH oxidase activity and in inducible nitric oxide synthase induction by aggregated Abeta1-42 in microglia. Glia. 2009;57:1727-40.

14. Lee M, McGeer E, McGeer PL. Activated human microglia stimulate neuroblastoma cells to upregulate production of beta amyloid protein and tau: implications for Alzheimer's disease pathogenesis. Neurobiol Aging. 2015;36:42-52

15. Giacobini E, Gold G. Alzheimer disease therapy-moving from amyloid-beta to tau. Nat Rev Neurol. 2013;9:677-86

16. Bayer TA, Wirths O. Focusing the amyloid cascade hypothesis on $\mathrm{N}$-truncated Abeta peptides as drug targets against Alzheimer's disease. Acta Neuropathol. 2014;127:787-801.

17. Wisniewski T, Goni F. Immunotherapeutic approaches for Alzheimer's disease. Neuron. 2015;85:1162-76.

18. Lemere CA. Immunotherapy for Alzheimer's disease: hoops and hurdles. Mol Neurodegener. 2013;8:36

19. Panza F, Logroscino G, Imbimbo BP, Solfrizzi V. Is there still any hope for amyloid-based immunotherapy for Alzheimer's disease? Curr Opin Psychiatry. 2014;27:128-37.

20. Zotova E, Holmes C, Johnston D, Neal JW, Nicoll JA, Boche D. Microglial alterations in human Alzheimer's disease following Abeta42 immunization. Neuropathol Appl Neurobiol. 2011;37:513-24.

21. Zotova E, Bharambe V, Cheaveau M, Morgan W, Holmes C, Harris S, Neal JW, Love S, Nicoll JA, Boche D. Inflammatory components in human Alzheimer's disease and after active amyloid-beta42 immunization. Brain. 2013;136:2677-96.

22. Gilman S, Koller M, Black RS, Jenkins L, Griffith SG, Fox NC, Eisner L, Kirby L, Rovira MB, Forette F, et al. Clinical effects of Abeta immunization (AN1792) in patients with AD in an interrupted trial. Neurology. 2005;64:1553-62.

23. Paquet C, Amin J, Mouton-Liger F, Nasser M, Love S, Gray F, Pickering RM, Nicoll JA, Holmes C, Hugon J, Boche D. Effect of active Abeta immunotherapy on neurons in human Alzheimer's disease. J Pathol. 2015;235:721-30

24. Rubio-Perez JM, Morillas-Ruiz JM. A review: inflammatory process in Alzheimer's disease, role of cytokines. ScientificWorldJournal. 2012;2012:756357.

25. McGeer PL, McGeer EG. Targeting microglia for the treatment of Alzheimer's disease. Expert Opin Ther Targets. 2015;19:497-506.

26. Tang Y, Le W. Differential roles of M1 and M2 microglia in neurodegenerative diseases. Mol Neurobiol. 2015.

27. Zhang G, Guo D, Dash PK, Arainga M, Wiederin JL, Haverland NA, KnibbeHollinger J, Martinez-Skinner A, Ciborowski P, Goodfellow VS, et al The mixed lineage kinase-3 inhibitor URMC-099 improves therapeutic outcomes for long-acting antiretroviral therapy. Nanomedicine. 2015.

28. Marker DF, Tremblay ME, Puccini JM, Barbieri J, Gantz Marker MA, Loweth CJ, Muly EC, Lu SM, Goodfellow VS, Dewhurst S, Gelbard HA. The new small-molecule mixed-lineage kinase 3 inhibitor URMC-099 is neuroprotective and anti-inflammatory in models of human immunodeficiency virus-associated neurocognitive disorders. J Neurosci. 2013;33:9998-10010.

29. Goodfellow VS, Loweth CJ, Ravula SB, Wiemann T, Nguyen T, Xu Y, Todd DE, Sheppard D, Pollack S, Polesskaya O, et al. Discovery, synthesis, and characterization of an orally bioavailable, brain penetrant inhibitor of mixed lineage kinase 3. J Med Chem. 2013;56:8032-48.

30. Floden AM, Li S, Combs CK. Beta-amyloid-stimulated microglia induce neuron death via synergistic stimulation of tumor necrosis factor alpha and NMDA receptors. J Neurosci. 2005;25:2566-75.

31. Kiyota T, Yamamoto M, Xiong H, Lambert MP, Klein WL, Gendelman HE, Ransohoff RM, Ikezu T. CCL2 accelerates microglia-mediated Abeta oligomer formation and progression of neurocognitive dysfunction. PLoS One. 2009:4, e6197.

32. Kiyota T, Gendelman HE, Weir RA, Higgins EE, Zhang G, Jain M. CCL2 affects beta-amyloidosis and progressive neurocognitive dysfunction in a mouse model of Alzheimer's disease. Neurobiol Aging. 2013;34:1060-8.

33. Kiyota T, Zhang G, Morrison CM, Bosch ME, Weir RA, Lu Y, Dong W, Gendelman HE. AAV2/1 CD74 gene transfer reduces beta-amyloidosis and improves learning and memory in a mouse model of Alzheimer's disease. Mol Ther. 2015;23:1712-21.

34. Yamamoto M, Kiyota T, Walsh SM, Liu J, Kipnis J, Ikezu T. Cytokine-mediated inhibition of fibrillar amyloid-beta peptide degradation by human mononuclear phagocytes. J Immunol. 2008;181:3877-86.

35. Wang X, Spandidos A, Wang H, Seed B. PrimerBank: a PCR primer database for quantitative gene expression analysis, 2012 update. Nucleic Acids Res. 2012:40:D1144-9.

36. Kim SH, Smith CJ, Van Eldik LJ. Importance of MAPK pathways for microglial pro-inflammatory cytokine IL-1 beta production. Neurobiol Aging. 2004:25:431-9.

37. Franciosi S, Ryu JK, Choi HB, Radov L, Kim SU, McLarnon JG. Broad-spectrum effects of 4-aminopyridine to modulate amyloid beta1-42-induced cell signaling and functional responses in human microglia. J Neurosci. 2006;26:11652-64

38. Sondag CM, Dhawan G, Combs CK. Beta amyloid oligomers and fibrils stimulate differential activation of primary microglia. J Neuroinflammation. 2009:6:1.

39. Xu Y, Hou XY, Liu Y, Zong YY. Different protection of K252a and $\mathrm{N}$-acetyl-L-cysteine against amyloid-beta peptide-induced cortical neuron apoptosis involving inhibition of MLK3-MKK7-JNK3 signal cascades. J Neurosci Res. 2009;87:918-27.

40. Gallo KA, Johnson GL. Mixed-lineage kinase control of JNK and p38 MAPK pathways. Nat Rev Mol Cell Biol. 2002;3:663-72.

41. Takata K, Kitamura Y, Saeki M, Terada M, Kagitani S, Kitamura R, Fujikawa Y, Maelicke A, Tomimoto H, Taniguchi T, Shimohama S. Galantamine-induced amyloid-\{beta\} clearance mediated via stimulation of microglial nicotinic acetylcholine receptors. J Biol Chem. 2010;285:40180-91.

42. Koenigsknecht-Talboo J, Landreth GE. Microglial phagocytosis induced by fibrillar beta-amyloid and IgGs are differentially regulated by proinflammatory cytokines. J Neurosci. 2005;25:8240-9.

43. Goerdt $\mathrm{S}$, Orfanos CE. Other functions, other genes: alternative activation of antigen-presenting cells. Immunity. 1999;10:137-42.

44. Zelcer N, Khanlou N, Clare R, Jiang Q, Reed-Geaghan EG, Landreth GE, Vinters HV, Tontonoz P. Attenuation of neuroinflammation and Alzheimer's disease pathology by liver $x$ receptors. Proc Natl Acad Sci U S A. 2007;104:10601-6.

45. Hickman SE, Allison EK, El Khoury J. Microglial dysfunction and defective beta-amyloid clearance pathways in aging Alzheimer's disease mice. J Neurosci. 2008;28:8354-60.

46. Fiala M, Cribbs DH, Rosenthal M, Bernard G. Phagocytosis of amyloid-beta and inflammation: two faces of innate immunity in Alzheimer's disease. J Alzheimers Dis. 2007;11:457-63.

47. Heneka MT, Rodriguez JJ, Verkhratsky A. Neuroglia in neurodegeneration. Brain Res Rev. 2010;63:189-211

48. Hanisch UK, Kettenmann H. Microglia: active sensor and versatile effector cells in the normal and pathologic brain. Nat Neurosci. 2007;10:1387-94.

49. Lee $C Y$, Landreth GE. The role of microglia in amyloid clearance from the AD brain. J Neural Transm (Vienna). 2010;117:949-60.

50. Perry VH, Nicoll JA, Holmes C. Microglia in neurodegenerative disease. Nat Rev Neurol. 2010;6:193-201.

51. Hardy J, Selkoe DJ. The amyloid hypothesis of Alzheimer's disease: progress and problems on the road to therapeutics. Science. 2002;297:353-6. 
52. Morgan D. Immunotherapy for Alzheimer's disease. J Intern Med. 2011;269:54-63.

53. Citron M. Alzheimer's disease: strategies for disease modification. Nat Rev Drug Discov. 2010;9:387-98.

54. Doraiswamy PM, Xiong GL. Pharmacological strategies for the prevention of Alzheimer's disease. Expert Opin Pharmacother. 2006;7:1-10.

55. Maioli E, Torricelli C, Valacchi G. Rottlerin and curcumin: a comparative analysis. Ann N Y Acad Sci. 2012;1259:65-76.

56. Wang LH, Besirli CG, Johnson Jr EM. Mixed-lineage kinases: a target for the prevention of neurodegeneration. Annu Rev Pharmacol Toxicol. 2004;44:451-74.

57. Gelbard HA, Dewhurst S, Maggirwar SB, Kiebala M, Polesskaya O, Gendelman HE. Rebuilding synaptic architecture in HIV-1 associated neurocognitive disease: a therapeutic strategy based on modulation of mixed lineage kinase. Neurotherapeutics. 2010;7:392-8.

58. Wang MJ, Huang HY, Chen WF, Chang HF, Kuo JS. Glycogen synthase kinase-3beta inactivation inhibits tumor necrosis factor-alpha production in microglia by modulating nuclear factor kappaB and MLK3/JNK signaling cascades. J Neuroinflammation. 2010;7:99.

59. Maroney AC, Finn JP, Connors TJ, Durkin JT, Angeles T, Gessner G, Xu Z, Meyer SL, Savage MJ, Greene LA, et al. Cep-1347 (KT7515), a semisynthetic inhibitor of the mixed lineage kinase family. J Biol Chem. 2001;276:25302-8.

60. Jaeschke A, Davis RJ. Metabolic stress signaling mediated by mixed-lineage kinases. Mol Cell. 2007;27:498-508.

61. Ajmone-Cat MA, Mancini M, De Simone R, Cilli P, Minghetti L. Microglial polarization and plasticity: evidence from organotypic hippocampal slice cultures. Glia. 2013:61:1698-711.

62. Gordon S. Alternative activation of macrophages. Nat Rev Immunol. 2003:3:23-35.

63. Ferrante CJ, Leibovich SJ. Regulation of macrophage polarization and wound healing. Adv Wound Care (New Rochelle). 2012;1:10-6.

64. Varnum MM, Ikezu T. The classification of microglial activation phenotypes on neurodegeneration and regeneration in Alzheimer's disease brain. Arch Immunol Ther Exp (Warsz). 2012;60:251-66.

65. Yu Y, Ye RD. Microglial Abeta receptors in Alzheimer's disease. Cell Mol Neurobiol. 2015;35:71-83.

66. Kouadir M, Yang L, Tu J, Yin X, Zhou X, Zhao D. Comparison of mRNA expression patterns of class B scavenger receptors in BV2 microglia upon exposure to amyloidogenic fragments of beta-amyloid and prion proteins. DNA Cell Biol. 2011;30:893-7.

67. Bamberger ME, Harris ME, McDonald DR, Husemann J, Landreth GE. A cell surface receptor complex for fibrillar beta-amyloid mediates microglial activation. J Neurosci. 2003;23:2665-74.

68. Koenigsknecht J, Landreth G. Microglial phagocytosis of fibrillar beta-amyloid through a beta1 integrin-dependent mechanism. J Neurosci. 2004;24:9838-46.

69. Wilkinson B, Koenigsknecht-Talboo J, Grommes C, Lee CY, Landreth G. Fibrillar beta-amyloid-stimulated intracellular signaling cascades require Vav for induction of respiratory burst and phagocytosis in monocytes and microglia. J Biol Chem. 2006;281:20842-50.

\section{Submit your next manuscript to BioMed Central and we will help you at every step:}

- We accept pre-submission inquiries

- Our selector tool helps you to find the most relevant journal

- We provide round the clock customer support

- Convenient online submission

- Thorough peer review

- Inclusion in PubMed and all major indexing services

- Maximum visibility for your research

Submit your manuscript at www.biomedcentral.com/submit 